\title{
A Note on Weak Type $(1,1)$ Estimate for the Higher Order Commutators of Christ-Journé Type
}

Yong Ding ${ }^{1}$ and Xudong Lai ${ }^{2 *}$

${ }^{1}$ School of Mathematical Sciences, Beijing Normal University, Beijing 100875, China

2 Institute for Advanced Study in Mathematics, Harbin Institute of Technology,

Harbin 150001, China

Received 12 December 2017; Accepted (in revised version) 21 January 2018

Abstract. In this paper, a weak type $(1,1)$ estimate is established for the higher order commutator introduced by Christ and Journé which is defined by

$$
T\left[a_{1}, \cdots, a_{l}\right] f(x)=\text { p.v. } \int_{\mathbb{R}^{d}} K(x-y)\left(\prod_{i=1}^{l} m_{x, y} a_{i}\right) \cdot f(y) d y,
$$

where $K$ is the standard Calderón-Zygmund convolution kernel on $\mathbb{R}^{d}(d \geq 2)$ and $m_{x, y} a_{i}=\int_{0}^{1} a_{i}(s x+(1-s) y) d s$.

Key Words: Weak type $(1,1)$, higher order, commutator.

AMS Subject Classifications: 42B20, 42B25

\section{Introduction}

Suppose that $K$ is the standard Calderón-Zygmund convolution kernel on $\mathbb{R}^{d} \backslash\{0\},(d \geq$ 2 ), which means that $K$ satisfies the following conditions:

$$
\begin{aligned}
& |K(x)| \leq C|x|^{-d}, \quad \int_{R<|x|<2 R} K(x) d x=0 \text { holds for all } R>0, \\
& |K(x-y)-K(x)| \leq C|y|^{\delta}|x|^{-d-\delta} \text { for some } 0<\delta \leq 1 \text { if }|x|>2|y| .
\end{aligned}
$$

In 1987, Christ and Journé [5] introduced a higher dimensional commutator associated with $K$ and $a_{i} \in L^{\infty}\left(\mathbb{R}^{d}\right)(i=1, \cdots, l)$ by

$$
T\left[a_{1}, \cdots, a_{l}\right] f(x)=\text { p.v. } \int_{\mathbb{R}^{d}} K(x-y)\left(\prod_{i=1}^{l} m_{x, y} a_{i}\right) \cdot f(y) d y, \quad f \in \mathcal{S}\left(\mathbb{R}^{d}\right),
$$

*Corresponding author. Email addresses: dingy@bnu. edu.cn (Y. Ding), xudonglai@hit.edu.cn (X. D. Lai) 
where $\mathcal{S}\left(\mathbb{R}^{d}\right)$ denotes the Schwartz class and

$$
m_{x, y} a_{i}=\int_{0}^{1} a_{i}((1-t) x+t y) d t=\int_{0}^{1} a_{i}(t x+(1-t) y) d t
$$

Note that $T\left[a_{1}, \cdots, a_{l}\right] f(x)$ can be seen as a higher dimensional generalization of the following commutator

$$
\text { p.v. } \int_{\mathbb{R}} \prod_{i=1}^{l}\left(\frac{A_{i}(x)-A_{i}(y)}{x-y}\right) \frac{f(y)}{x-y} d y,
$$

which is the famous Calderón commutator discussed in [3] and is related to the study of the Cauchy integral, boundary value problem of elliptic equation on non-smooth domain (see e.g., $[4,10,15])$.

Observe that the kernel $K(x-y)$ is smooth but $m_{x, y} a_{i}$ has no smoothness about variable $x$ and $y$ if $a_{i} \in L^{\infty}\left(\mathbb{R}^{d}\right)$. Therefore the standard Calderón-Zygmund theory cannot be applied directly. Christ and Journé [5] proved that $T\left[a_{1}, \cdots, a_{l}\right]$ is bounded on $L^{p}\left(\mathbb{R}^{d}\right)$ $(1<p<\infty)$ when $a_{i} \in L^{\infty}\left(\mathbb{R}^{d}\right)(i=1, \cdots, l)$. In 1995, Hofmann [14] gave the weighted $L^{p}\left(\mathbb{R}^{d}\right)$ $(1<p<\infty)$ boundedness of $T\left[a_{1}, \cdots, a_{l}\right]$, when the kernel $K(x)=\Omega(x /|x|)|x|^{-d}$. Recently, there are renew interests on this singular integral of Christ-Journé type since it has some direct applications in the mixing flows problem (see e.g., [2, 13]). In 2015, A. Seeger, C. Smart and B. Street [19] further studied the commutator of Christ-Journé type and established some multilinear estimates. Later, the second author of the present paper established all multilinear estimates of the higher Calderón commutator (see [16]). For the endpoint case $p=1$, the weak type $(1,1)$ estimate seems to be difficulty and the previous result is only known for the first order commutator. In 2012, Grafakos and Honzík [12] proved that the commutator $T[a]$ is of weak type $(1,1)$ for $d=2$. Later, Seeger [18] showed that $T[a]$ is also of weak type $(1,1)$ for all $d \geq 2$. In [6], the authors established weighted $L^{p}$ boundedness of $T[a]$ for $A_{p}$ weight with $d \geq 2$ and weighted weak type $(1,1)$ boundedness for power weight $|x|^{\alpha}(-2<\alpha<0)$ with $d=2$ (later we extended this result to general $A_{1}$ weight for all $d \geq 2$ in [8]). However, the weak type $(1,1)$ estimate for the higher order commutator seems to be unexplored and may be very difficult since the kernel involves with more than two rough factors $\prod_{i=1}^{l} m_{x, y} a_{i}$ under the condition that all $a_{i} \in L^{\infty}\left(\mathbb{R}^{d}\right)(i=1, \cdots, l)$. In this paper, we try to give a weak type $(1,1)$ estimate for $T\left[a_{1}, \cdots, a_{l}\right]$ with some restricted condition of $a_{i}$. Our main result is as follows.

Theorem 1.1. Suppose $K$ satisfies (1.1a) and (1.1b) for $d \geq 2$. Let $a_{1} \in L^{\infty}\left(\mathbb{R}^{d}\right)$. Assume $a_{i}, \widehat{a_{i}} \in$ $L^{1}\left(\mathbb{R}^{d}\right), i=2, \cdots, l$. Then there exists a constant $C>0$ such that

$$
m\left(\left\{x \in \mathbb{R}^{d}:\left|T\left[a_{1}, \cdots, a_{l}\right] f(x)\right|>\lambda\right\}\right) \leq C \lambda^{-1}\left\|a_{1}\right\|_{\infty}\left(\prod_{i=2}^{l}\left\|\widehat{a}_{i}\right\|_{1}\right)\|f\|_{1}
$$

for all $\lambda>0$ and $f \in L^{1}\left(\mathbb{R}^{d}\right)$. 
Remark 1.1. By using Fourier inversive formula, it is easy to see that the condition $a$, $\hat{a} \in L^{1}\left(\mathbb{R}^{d}\right)$ implies $a \in L^{\infty}\left(\mathbb{R}^{d}\right)$. To remove this kind of restricted condition, some new ideas may be needed.

When the dimension $d=2$, Grafakos and Honzík [12] used the $T T^{*}$ method to show that the first order commutator $T[a]$ is of weak type $(1,1)$. In [18], Seeger used the microlocal decomposition of the kernel and the Littlewood-Paley decomposition of $a$. However it seems to be difficult to use these ideas from [12] and [18] to deal with the higher order commutator which involves more than two rough factors. In the present paper, we add some restricted condition $\hat{a}_{i} \in L^{1}\left(\mathbb{R}^{d}\right)$ for $i=2, \cdots, l$. So we can make a modified CalderónZygmund decomposition of a function with some parameters come from $a_{2}, \cdots, a_{l}$, but those bounds in the Calderón-Zygmund decomposition are independent of these parameters (see Lemma 2.1). Applying this kind of Calderón-Zygmund decomposition, the kernel essentially has only one rough kernel under the restricted condition $\hat{a}_{i} \in L^{1}\left(\mathbb{R}^{d}\right)$ for $i=2, \cdots, l$. Then using some idea from $[7,9,17,18]$, we may get the weak type $(1,1)$ bound for the higher order commutator.

This paper is organized as follows. In Section 2, we complete the proof of Theorem 1.1 based on some lemmas, their proofs will be given in Section 3 and Section 4 . Throughout this paper, the letter $C$ stands for a positive constant which is independent of the essential variables and not necessarily the same one in each occurrence. $A \lesssim B$ means $A \leq C B$ for some constant $C$. $A \approx B$ means that $A \lesssim B$ and $B \lesssim A$. For a set $E \subset \mathbb{R}^{\widetilde{d}}$, we denote Lebesgue measure of $E$ by $|E|$ or $m(E)$. Denote by $\mathcal{F} f$ and $\hat{f}$ the Fourier transform of $f$, which is defined by

$$
\mathcal{F} f(\xi)=\int_{\mathbb{R}^{d}} e^{-i\langle x, \xi\rangle} f(x) d x
$$

$\mathbb{Z}_{+}$denote the set of all nonnegative integers and $\mathbb{Z}_{+}^{d}=\mathbb{Z}_{+} \times \cdots \times \mathbb{Z}_{+} \cdot[x]$ denotes the integer part of $x$.

\section{Proof of Theorem 1.1: setup}

In this section we give the proof of Theorem 1.1 based on some lemmas, their proofs will be given in Section 3 and Section 4, respectively.

Using the inversive Fourier formula under the condition that $a_{i}, \widehat{a}_{i} \in L^{1}\left(\mathbb{R}^{d}\right), i=2, \cdots, l$, we write each term

$$
m_{x, y} a_{i}=\frac{1}{(2 \pi)^{d}} \int_{0}^{1} \int_{\mathbb{R}^{d}} \widehat{a}_{i}\left(\eta_{i}\right) e^{i s_{i}\left\langle\eta_{i}, x\right\rangle} e^{i\left(1-s_{i}\right)\left\langle y, \eta_{i}\right\rangle} d \eta_{i} d s_{i}
$$


Therefore by Fubini's theorem, we have

$$
\begin{aligned}
T\left[a_{1}, \cdots, a_{l}\right] f(x)= & \frac{1}{(2 \pi)^{(l-1) d}} \text { p.v. } \int_{\mathbb{R}^{d}} K(x-y) m_{x, y} a_{1} \cdot f(y) \\
& \quad \times\left(\iint_{[0,1]^{l-1} \times\left(\mathbb{R}^{d}\right)^{l-1}} \prod_{i=2}^{l} \widehat{a}_{i}\left(\eta_{i}\right) e^{i s_{i}\left\langle x, \eta_{i}\right\rangle} e^{i\left(1-s_{i}\right)\left\langle y, \eta_{i}\right\rangle} d \vec{s} d \vec{\eta}\right) d y \\
= & \iint_{E^{l}} u^{\vec{\eta}, \vec{s}}(x)\left(T\left[a_{1}\right] W^{\vec{\eta}, \vec{s}} f\right)(x) d \vec{\eta} d \vec{s},
\end{aligned}
$$

where

$$
\begin{array}{ll}
E^{l}=[0,1]^{l-1} \times\left(\mathbb{R}^{d}\right)^{l-1}, & u^{\vec{\eta}, \vec{s}}(x)=(2 \pi)^{-(l-1) d} \prod_{i=2}^{l} \widehat{a}_{i}\left(\eta_{i}\right) e^{i s_{i}\left\langle x, \eta_{i}\right\rangle}, \\
d \vec{\eta}=d \eta_{2} \cdots d \eta_{l}, & W^{\vec{\eta}, \vec{s}}(y)=\prod_{i=2}^{l} e^{i\left(1-s_{i}\right)\left\langle y, \eta_{i}\right\rangle} \quad \text { and } \quad d \vec{s}=d s_{2} \cdots d s_{n} .
\end{array}
$$

In the following, we try to make a Calderón-Zygmund decomposition of $W^{\vec{\eta}, \vec{s}} f$ with the underlying cubes independent of $\vec{\eta}, \vec{s}$.

Lemma 2.1. Let $f \in L^{1}\left(\mathbb{R}^{d}\right)$ and $\lambda>0$. Set $\Omega_{\lambda}=\left\{x \in \mathbb{R}^{d}: M(f)(x)>\lambda\right\}$ where $M$ is the Hardy-Littlewood maximal operator. Then we have the following conclusions:

(i) $\Omega_{\lambda}=\bigcup Q, Q^{\prime}$ s are disjoint dyadic cubes. Let $Q$ be the collection of all these cubes.

(ii) $m\left(\Omega_{\lambda}\right) \lesssim \frac{1}{\lambda}\|f\|_{1}$.

(iii) $f W^{\vec{\eta}, \vec{s}}=g^{\vec{\eta}, \vec{s}}+b^{\vec{\eta}, \vec{s}}$.

(iv) $b^{\vec{\eta}, \vec{s}}=\sum_{Q} b_{Q}^{\vec{\eta}, \vec{s}}, \operatorname{supp} b_{Q}^{\vec{\eta}, \vec{s}} \subset Q, \int b_{Q}^{\vec{\eta}, \vec{s}}=0,\left\|b_{Q}^{\vec{\eta}, \vec{s}}\right\|_{1} \lesssim \lambda|Q|,\left\|b^{\vec{\eta}, \vec{s}}\right\|_{1} \lesssim\|f\|_{1}$.

(v) $\left\|g^{\vec{\eta}, \vec{s}}\right\|_{2}^{2} \lesssim \lambda\|f\|_{1}$.

All the explicit constants that appear in (i)-(v) above are independent of $\vec{\eta}, \vec{s}$.

Proof. We first make a Whitney decomposition of the set $\Omega_{\lambda}$. Then there exists a family of dyadic closed cubes $\left\{Q_{j}\right\}_{j}$ (e.g., see [11]) such that

(a) $\Omega_{\lambda}=\bigcup Q_{j}$ and $Q_{j}{ }^{\prime}$ s have disjoint interior.

(b) $\sqrt{d} \cdot l\left(Q_{j}\right) \leq \operatorname{dist}\left(Q_{j}, \Omega_{\lambda}^{c}\right) \leq 4 \sqrt{d} \cdot l\left(Q_{j}\right)$, where $l\left(Q_{j}\right)$ denotes the side's length of $Q_{j}$.

By the weak type $(1,1)$ of $M$, we have

$$
m\left(\Omega_{\lambda}\right) \lesssim \frac{1}{\lambda}\|f\|_{1}
$$

We write $f W^{\vec{\eta}, \vec{s}}=g^{\vec{\eta}, \vec{s}}+b^{\vec{\eta}, \vec{s}}$, where

$$
\begin{aligned}
& g^{\vec{\eta}, \vec{s}}=f W^{\vec{\eta}, \vec{s}} \chi_{\Omega_{\lambda}^{c}}+\sum_{Q}\left(\frac{1}{|Q|} \int_{Q} f(x) W^{\vec{\eta}, \vec{s}}(x) d x\right) \chi_{Q}, \\
& b^{\vec{\eta}, \vec{s}}=\sum_{Q}\left\{f W^{\vec{\eta}, \vec{s}}-\frac{1}{|Q|} \int_{Q} f(x) W^{\vec{\eta}, \vec{s}}(x) d x\right\} \chi_{Q}=: \sum_{Q} b_{Q}^{\vec{\eta}, \vec{s}} .
\end{aligned}
$$


So, $b_{Q}^{\vec{\eta}, \vec{s}}$ is supported in $Q$ and $\int b_{Q}^{\vec{\eta}, \vec{s}}=0$. Let $t Q$ denote the cube with $t$ times the side length of $Q$ and the same center. We now claim that

$$
\frac{1}{|Q|} \int_{Q}|f(x)| d x \lesssim \lambda
$$

In fact, by the Whitney decomposition's property (b) we have $9 \sqrt{d} Q \cap \Omega_{\lambda}^{c} \neq \varnothing$. Thus by the definition of $\Omega_{\lambda}^{c}$, there exists $x_{0} \in 9 \sqrt{d} Q$ such that $M f\left(x_{0}\right) \leq \lambda$. Using the property of the maximal function, we have $\frac{1}{|9 \sqrt{d} Q|} \int_{9 \sqrt{d} Q}|f(x)| d x \lesssim \lambda$. Hence we conclude that

$$
\frac{1}{|Q|} \int_{Q}|f(x)| d x \leq \frac{1}{|Q|} \int_{9 \sqrt{d} Q}|f(x)| d x \lesssim \lambda .
$$

For $b_{Q}^{\vec{\eta}, \vec{s}}$ and $b^{\vec{\eta}, \vec{s}}$, by (2.2) and (2.3) we have

$$
\left\|b_{Q}^{\vec{\eta}, \vec{s}}\right\|_{1} \leq 2 \int_{Q}|f(x)| d x \lesssim \lambda|Q|, \quad\left\|b^{\vec{\eta}, \vec{s}}\right\|_{1} \lesssim\|f\|_{1}+\lambda m\left(\Omega_{\lambda}\right) \lesssim\|f\|_{1} .
$$

Note that $|f(x)| \leq \lambda$ almost everywhere in $\Omega_{\lambda}^{c}$. Using this fact, (2.2) and (2.3), we have

$$
\left\|g^{\vec{\eta}, \vec{s}}\right\|_{2}^{2} \lesssim \lambda\|f\|_{1}+\lambda^{2} m\left(\Omega_{\lambda}\right) \lesssim \lambda\|f\|_{1}
$$

Thus, we complete the proof.

Now we set up the proof of Theorem 1.1 with a series of lemmas. We only focus on dimension $d \geq 2$. By using scaling arguments, we may assume $\left\|a_{1}\right\|_{\infty}=\left\|\widehat{a_{i}}\right\|_{1}=1, i=2, \cdots, l$.

By (2.1) and the property (iii) in Lemma 2.1, we obtain

$$
\begin{aligned}
\left|\left\{x:\left|T\left[a_{1}, \cdots, a_{l}\right] f(x)\right|>\lambda\right\}\right| \leq & m\left(\left\{x:\left|\iint_{E^{l}} u^{\vec{\eta}, \vec{s}}(x) T\left[a_{1}\right] g^{\vec{\eta}, \vec{s}}(x) d \vec{\eta} d \vec{s}\right|>\lambda / 2\right\}\right) \\
& +m\left(\left\{x:\left|\iint_{E^{l}} u^{\vec{\eta}, \vec{s}}(x) T\left[a_{1}\right] b^{\vec{\eta}, \vec{s}}(x) d \vec{\eta} d \vec{s}\right|>\lambda / 2\right\}\right) .
\end{aligned}
$$

Hence, using Chebyshev's inequality, the fact $T\left[a_{1}\right]$ is bounded on $L^{2}\left(\mathbb{R}^{d}\right)$ with bound $C\left\|a_{1}\right\|_{\infty}$ (see [5]) and the property (iv) in Lemma 2.1, we get

$$
\begin{aligned}
&\left|\left\{x \in \mathbb{R}^{d}: \iint_{E^{l}} u^{\vec{\eta}, \vec{s}}(x) T\left[a_{1}\right] g^{\vec{\eta}, \vec{s}}(x) d \vec{\eta} d \vec{s} \mid>\lambda / 2\right\}\right| \\
& \lesssim \lambda^{-2}\left\|\iint_{E^{l}} u^{\vec{\eta}, \vec{s}}(x) T\left[a_{1}\right] g^{\vec{\eta}, \vec{s}}(x) d \vec{\eta} d \vec{s}\right\|_{2}^{2} \\
& \lesssim \lambda^{-2}\left(\iint_{E^{l}}\left(\prod_{i=2}^{l}\left|\widehat{a_{i}\left(\eta_{i}\right)}\right|\right)\left\|T\left[a_{1}\right] g^{\vec{\eta}, \vec{s}}(x)\right\|_{2} d \vec{\eta} d \vec{s}\right)^{2} \\
& \lesssim \lambda^{-2}\left(\iint_{E^{l}}\left(\prod_{i=2}^{l}\left|\widehat{a_{i}\left(\eta_{i}\right)}\right|\right)\left\|a_{1}\right\|_{\infty}\left\|g^{\vec{\eta}, \vec{s}}\right\|_{2} d \vec{\eta} d \vec{s}\right)^{2} \\
& \lesssim \lambda^{-1}\|f\|_{1} .
\end{aligned}
$$


Define $E^{*}=\bigcup_{Q \in Q} 2^{200} Q$. Then we have

$$
\begin{aligned}
& m\left(\left\{x:\left|\iint_{E^{l}} u^{\vec{\eta}, \vec{s}}(x) T\left[a_{1}\right] b^{\vec{\eta}, \vec{s}}(x) d \vec{\eta} d \vec{s}\right|>\lambda / 2\right\}\right) \\
\leq & m\left(E^{*}\right)+m\left(\left\{x \in\left(E^{*}\right)^{c}:\left|\iint_{E^{l}} u^{\vec{\eta}, \vec{s}}(x) T\left[a_{1}\right] b^{\vec{\eta}, \vec{s}}(x) d \vec{\eta} d \vec{s}\right|>\lambda / 2\right\}\right) .
\end{aligned}
$$

By the property (ii) in Lemma 2.1, the set $E^{*}$ satisfies

$$
m\left(E^{*}\right) \lesssim m\left(\Omega_{\lambda}\right) \lesssim \lambda^{-1}\|f\|_{1}
$$

Thus, to finish the proof of Theorem 1.1, it suffices to show

$$
m\left(\left\{x \in\left(E^{*}\right)^{c}:\left|\iint_{E^{l}} u^{\vec{\eta}, \vec{s}}(x) T\left[a_{1}\right] b^{\vec{\eta}, \vec{s}}(x) d \vec{\eta} d \vec{s}\right|>\lambda / 2\right\}\right) \lesssim \frac{\|f\|_{1}}{\lambda} .
$$

Denote $\mathfrak{Q}_{k}=\left\{Q \in \mathcal{Q}: l(Q)=2^{k}\right\}$ and let $\mathfrak{B}_{k}^{\vec{\eta}, \vec{s}}=\sum_{Q \in \mathfrak{Q}_{k}} b_{Q}^{\vec{\eta}, \vec{s}}$. Then $b^{\vec{\eta}, \vec{s}}$ can be rewritten as $b^{\vec{\eta}, \vec{s}}=\sum_{j \in \mathbb{Z}} \mathfrak{B}_{j}^{\vec{\eta}, \vec{s}}$. Let $\psi$ be a radial $C^{\infty}$ function such that $\psi(\xi)=1$ for $|\xi| \leq 1, \psi(\xi)=0$ for $|\xi| \geq 2$ and $0 \leq \psi(\xi) \leq 1$ for all $\xi \in \mathbb{R}^{d}$. Define $\phi(x)=\psi(x)-\psi(2 x)$. Then supp $\phi \subset\left\{x: \frac{1}{2} \leq|x| \leq 2\right\}$ and $\sum_{j} \phi_{j}(x)=1$ for all $x \in \mathbb{R}^{d} \backslash\{0\}$, where $\phi_{j}(x)=\phi\left(2^{-j} x\right)$. Now we define the operator $T_{j}\left[a_{1}\right]$ as

$$
T_{j}\left[a_{1}\right] f(x)=\int_{\mathbb{R}^{d}} \phi_{j}(x-y) K(x-y) m_{x, y} a_{1} \cdot f(y) d y .
$$

Then $T\left[a_{1}\right]=\sum_{j} T_{j}\left[a_{1}\right]$. For simplicity, we set $K_{j}(x)=\phi_{j}(x) K(x)$. We write

$$
T\left[a_{1}\right] b^{\vec{\eta}, \vec{s}}(x)=\sum_{n \in \mathbb{Z}} \sum_{j \in \mathbb{Z}} T_{j}\left[a_{1}\right] \mathfrak{B}_{j-n}^{\vec{\eta}, \vec{s}} .
$$

Note that $T_{j}\left[a_{1}\right] \mathfrak{B}_{j-n}^{\vec{\eta}, \vec{s}}(x)=0$ for $x \in\left(E^{*}\right)^{c}$ and $n<100$. Therefore we only consider $n \geq 100$. Here we should point out that the number 100 is not very important. In fact, it is sufficient to consider large $n$ once we choose the set $E^{*}$ as large as we want. Write

$$
\begin{aligned}
& m\left(\left\{x \in\left(E^{*}\right)^{c}:\left|\iint_{E^{l}} u^{\vec{\eta}, \vec{s}}(x) T\left[a_{1}\right] b^{\vec{\eta}, \vec{s}}(x) d \vec{\eta} d \vec{s}\right|>\lambda / 2\right\}\right) \\
= & m\left(\left\{x \in\left(E^{*}\right)^{c}:\left|\iint_{E^{l}} u^{\vec{\eta}, \vec{s}}(x) \sum_{n \geq 100} \sum_{j \in \mathbb{Z}} T_{j}\left[a_{1}\right] \mathfrak{B}_{j-n}^{\vec{\eta}, \vec{s}}(x) d \vec{\eta} d \vec{s}\right|>\lambda / 2\right\}\right) .
\end{aligned}
$$

Hence, to finish the proof of of Theorem 1.1, it suffices to verify the following estimate:

$$
m\left(\left\{x \in \mathbb{R}^{d}:\left|\iint_{E^{l}} u^{\vec{\eta}, \vec{s}}(x) \sum_{n \geq 100} \sum_{j \in \mathbb{Z}} T_{j}\left[a_{1}\right] \mathfrak{B}_{j-n}^{\vec{\eta}, \vec{s}}(x) d \vec{\eta} d \vec{s}\right|>\lambda / 2\right\}\right) \lesssim \frac{\|f\|_{1}}{\lambda} .
$$




\subsection{Some key estimates}

Some important estimates play a key role in the proof of (2.6). We present them by some lemmas, which will be proved in Section 3 and Section 4. The first estimate tells us that the operator $T_{j}\left[a_{1}\right]$ can be approximated by an operator $T_{j}^{n}\left[a_{1}\right]$ in measure, which is defined below.

Let $l_{\tau}(n)=\tau \delta^{-1} n+2$, where $\tau>\frac{1}{n}>0$ and $0<\tau \delta^{-1}<1$ will be chosen later. As we mention before, we only need to consider sufficient larger $n$, so the constant $\tau$ could be chosen as small as want and it will be chosen at the end of this paper. Let $\eta$ be a nonnegative, radial $C^{\infty}$ function which is supported in $\{|x| \leq 1\}$ and satisfies $\int_{\mathbb{R}^{d}} \eta(x) d x=$ 1. Set $\eta_{i}(x)=2^{-i d} \eta\left(2^{-i} x\right)$. Define the operator $P_{t}$ by $P_{t} f(x)=\eta_{t} * f(x)$. Set

$$
K_{j}^{n}(x)=P_{j-l_{\tau}(n)} K_{j}(x) .
$$

Since $K_{j}(x)$ is supported in $\left\{2^{j-1} \leq|x| \leq 2^{j+1}\right\}$ and $\eta_{j-l_{\tau}(n)}(x)$ is supported in $\{|x| \leq$ $\left.2^{j-l_{\tau}(n)}\right\}$, we see that $K_{j}^{n}(x)$ is supported in $\left\{2^{j-2} \leq|x| \leq 2^{j+2}\right\}$. Therefore

$$
\left|K_{j}^{n}(x)\right| \lesssim 2^{-j d} \chi_{\left\{2^{j-2} \leq|x| \leq 2^{j+2}\right\}}
$$

and similarly for multi-indices $\alpha$,

$$
\left|\partial^{\alpha} K_{j}^{n}(x)\right| \lesssim 2^{-j d+\left(l_{\tau}(n)-j\right)|\alpha|} \chi_{\left\{2^{j-2} \leq|x| \leq 2^{j+2}\right\}} .
$$

Let $\rho_{n}$ be a smooth, nonnegative function such that $\rho_{n}(s)=1$ on $\left[2^{-\tau n}, 1-2^{-\tau n}\right]$, supp $\rho_{n} \subset$ $\left(2^{-\tau n-1}, 1-2^{-\tau n-1}\right)$, and the derivatives of $\rho_{n}$ satisfy the natural estimates

$$
\left|\frac{d^{k}}{d s^{k}} \rho_{n}(s)\right| \lesssim 2^{k \tau n} \quad \text { for all } k \in \mathbb{Z}_{+} \text {. }
$$

Let

$$
m_{x, y}^{n} a_{1}=\int_{0}^{1} \rho_{n}(s) a_{1}(s x+(1-s) y) d s .
$$

Define the operator $T_{j}^{n}\left[a_{1}\right]$ by

$$
T_{j}^{n}\left[a_{1}\right] f(x)=\int_{\mathbb{R}^{d}} K_{j}^{n}(x-y) m_{x, y}^{n} a_{1} \cdot f(y) d y .
$$

Lemma 2.2. With those definitions above, we have

$m\left(\left\{x \in \mathbb{R}^{d}:\left|\iint_{E^{l}} u^{\vec{\eta}, \vec{s}}(x) \sum_{n \geq 100} \sum_{j \in \mathbb{Z}}\left(T_{j}\left[a_{1}\right] \mathfrak{B}_{j-n}^{\vec{\eta}, \vec{s}}(x)-T_{j}^{n}\left[a_{1}\right] \mathfrak{B}_{j-n}^{\vec{\eta}, \vec{s}}(x)\right) d \vec{\eta} d \vec{s}\right|>\lambda / 4\right\}\right) \lesssim \frac{\|f\|_{1}}{\lambda}$.

By Lemma 2.2, the proof of (2.6) now is reduced to verify the following estimate:

$$
m\left(\left\{x \in \mathbb{R}^{d}:\left|\iint_{E^{l}} u^{\vec{\eta}, \vec{s}}(x) \sum_{n \geq 100} \sum_{j \in \mathbb{Z}} T_{j}^{n}\left[a_{1}\right] \mathfrak{B}_{j-n}^{\vec{\eta}, \vec{s}}(x) d \vec{\eta} d \vec{s}\right|>\lambda / 4\right\}\right) \lesssim \frac{\|f\|_{1}}{\lambda} .
$$

Below we separate $T_{j}^{n}\left[a_{1}\right]$ as $P_{j-n \kappa} T_{j}^{n}\left[a_{1}\right]+\left(I-P_{j-n \kappa}\right) T_{j}\left[a_{1}\right]$, where $\kappa$ satisfies $0<\kappa<1$ which will be chosen later. For $P_{j-n \kappa} T_{j}^{n}\left[a_{1}\right]$, we have the following lemma. 
Lemma 2.3. With those definitions above, for $n \geq 100$, we have

$$
\left\|P_{j-n \kappa} T_{j}^{n}\left[a_{1}\right] \mathfrak{B}_{j-n}^{\vec{\eta}, \vec{s}}\right\|_{1} \lesssim n\left(2^{-(1-\kappa) n}+2^{-\left(1-\tau \delta^{-1}\right) n}\right)\left\|\mathfrak{B}_{j-n}^{\vec{\eta}, \vec{s}}\right\|_{1} .
$$

Applying Chebyshev's inequality, Minkowski's inequality and Lemma 2.3 and the property (iv) in Lemma 2.1, one may get

$$
\begin{aligned}
& m\left(\left\{x \in \mathbb{R}^{d}:\left|\iint_{E^{l}} u^{\vec{\eta}, \vec{s}}(x) \sum_{n \geq 100 j \in \mathbb{Z}} \sum_{j-n \kappa} T_{j}^{n}\left[a_{1}\right] \mathfrak{B}_{j-n}^{\vec{\eta}, \vec{s}}(x) d \vec{\eta} d \vec{s}\right|>\lambda / 4\right\}\right) \\
\lesssim & \lambda^{-1} \iint_{E^{l}} \sum_{n \geq 100} \sum_{j}\left|\prod_{i=2}^{l} \hat{a}_{i}\left(\eta_{i}\right)\right|\left\|P_{j-n \kappa} T_{j}^{n}\left[a_{1}\right] \mathfrak{B}_{j-n}^{\vec{\eta}, \vec{s}}\right\|_{1} d \vec{\eta} d \vec{s} \\
\lesssim & \lambda^{-1} \sum_{n \geq 100} n\left(2^{(-1+\kappa) n}+2^{\left(-1+\tau \delta^{-1}\right) n}\right)\|f\|_{1} \lesssim \lambda^{-1}\|f\|_{1} .
\end{aligned}
$$

Now the problem is reduced to prove the estimate below

$$
m\left(\left\{x \in \mathbb{R}^{d}:\left|\iint_{E^{l}} u^{\vec{\eta}, \vec{s}}(x) \sum_{n \geq 100} \sum_{j \in \mathbb{Z}}\left(I-P_{j-n \kappa}\right) T_{j}^{n}\left[a_{1}\right] \mathfrak{B}_{j-n}^{\vec{\eta}, \vec{s}}(x) d \vec{\eta} d \vec{s}\right|>\lambda / 4\right\}\right) \lesssim \frac{\|f\|_{1}}{\lambda} .
$$

In the following, we need to make a microlocal decomposition of the kernel. To do this, we need to give a partition of unity on the unit surface $S^{d-1}$. Choose $n \geq 100$. Let $\Theta_{n}=$ $\left\{e_{v}^{n}\right\}_{v}$ be a collection of unit vectors on $S^{d-1}$ which satisfies the following two conditions:

(a) $\left|e_{v}^{n}-e_{v^{\prime}}^{n}\right| \geq 2^{-n \gamma-4}$, if $v \neq v^{\prime}$;

(b) If $\theta \in \mathbb{S}^{d-1}$, there exists an $e_{v}^{n}$ such that $\left|e_{v}^{n}-\theta\right| \leq 2^{-n \gamma-4}$.

The constant $\gamma$ in (a) and (b) satisfying $0<\tau \delta^{-1}<\gamma<\kappa<1$ which will be chosen later. In fact, we may simply take a maximal collection $\left\{e_{v}^{n}\right\}_{v}$ for which (a) holds. Notice that there are $C 2^{n \gamma(d-1)}$ elements in the collection $\left\{e_{v}^{n}\right\}_{v}$. For every $\theta \in \mathbb{S}^{d-1}$, there only exists finite $e_{v}^{n}$ such that $\left|e_{v}^{n}-\theta\right| \leq 2^{-n \gamma-4}$. Now we can construct an associated partition of unity on the unit surface $S^{d-1}$. Let $\zeta$ be a smooth, nonnegative, radial function with $\zeta(u)=1$ for $|u| \leq \frac{1}{2}$ and $\zeta(u)=0$ for $|u|>1$. Define

$$
\Psi_{v}^{n}(\xi)=\zeta\left(2^{n \gamma}\left(\frac{\xi}{|\xi|}-e_{v}^{n}\right)\right), \quad \Gamma_{v}^{n}(\xi)=\Psi_{v}^{n}(\xi)\left(\sum_{e_{v}^{n} \in \Theta_{n}} \Psi_{v}^{n}(\xi)\right)^{-1} .
$$

Then it is easy to see that $\Gamma_{v}^{n}$ is homogeneous of degree 0 with $\sum_{v} \Gamma_{v}^{n}(\xi)=1$ holds for all $\xi \neq 0$ and all $n$. In addition, we have the following estimate for multi-indices $\alpha$ and $\xi \neq 0$,

$$
\left|\partial_{\xi}^{\alpha} \Gamma_{v}^{n}(\xi)\right| \lesssim 2^{n \gamma|\alpha|}|\xi|^{-|\alpha|}
$$

Now we define operator $T_{j}^{n, v}\left[a_{1}\right]$ by

$$
T_{j}^{n, v}\left[a_{1}\right] f(x)=\int_{\mathbb{R}^{d}} K_{j}^{n, v}(x-y) m_{x, y}^{n} a_{1} \cdot f(y) d y,
$$


where $K_{j}^{n, v}(x)=K_{j}^{n}(x) \Gamma_{v}^{n}(x)$. Therefore, we have

$$
T_{j}^{n}\left[a_{1}\right]=\sum_{v} T_{j}^{n, v}\left[a_{1}\right]
$$

In the sequel, we need to sperate the phase of the frequent space into different direction. Hence we define a multiplier operator by

$$
\widehat{G_{n, v} h}(\xi)=\Phi\left(2^{n \gamma}\left\langle e_{v}^{n}, \xi /|\xi|\right\rangle\right) \hat{h}(\xi),
$$

where $h$ is a Schwartz function and $\Phi$ is a smooth, nonnegative, radial function such that $0 \leq \Phi(x) \leq 1$ and $\Phi(x)=1$ on $|x| \leq 2, \Phi(x)=0$ on $|x|>4$. Now let $G_{n, v}+\left(I-G_{n, v}\right)$ act on $T_{j}^{n, v}\left[a_{1}\right]$. Then we can split $T_{j}^{n, v}\left[a_{1}\right]$ into two parts:

$$
T_{j}^{n, v}\left[a_{1}\right]=G_{n, v} T_{j}^{n, v}\left[a_{1}\right]+\left(I-G_{n, v}\right) T_{j}^{n, v}\left[a_{1}\right] .
$$

The following lemmas give the $L^{2}$ estimate involving the term $G_{n, v}$, which will be proved in next section.

Lemma 2.4. With those definitions above, for $n \geq 100$,

$$
\left\|\iint_{E^{l}} u^{\vec{\eta}, \vec{s}}(x) \sum_{j \in \mathbb{Z}} \sum_{v} G_{n, v}\left(I-P_{j-n \kappa}\right) T_{j}^{n, v}\left[a_{1}\right] \mathfrak{B}_{j-n}^{\vec{\eta}, \vec{s}}(x) d \vec{\eta} d \vec{s}\right\|_{2}^{2} \lesssim 2^{-n \gamma} \lambda\|f\|_{1} .
$$

The estimates of the terms involving $\left(I-G_{n, v}\right)\left(I-P_{j-n \kappa}\right) T_{j}^{n, v}\left[a_{1}\right]$ are more complicated. In Section 4, we shall prove the following lemma.

Lemma 2.5. We have

$$
\left\|\iint_{E^{l}} u^{\vec{\eta}, \vec{s}}(x) \sum_{n \geq 100} \sum_{j \in \mathbb{Z}} \sum_{v}\left(I-G_{n, v}\right)\left(I-P_{j-n \kappa}\right) T_{j}^{n, v}\left[a_{1}\right] \mathfrak{B}_{j-n}^{\vec{\eta}, \vec{s}}(x) d \vec{\eta} d \vec{s}\right\|_{1} \lesssim\|f\|_{1} .
$$

\subsection{Proof of Theorem 1.1}

We now complete the proof of (2.9). By Chebyshev's inequality, we have

$$
\begin{aligned}
& m\left(\left\{x \in\left(E^{*}\right)^{c}:\left|\iint_{E^{l}} u^{\vec{\eta}, \vec{s}}(x) \sum_{n \geq 100} \sum_{j \in \mathbb{Z}}\left(I-P_{j-n \kappa}\right) T_{j}^{n}\left[a_{1}\right] \mathfrak{B}_{j-n}^{\vec{\eta}, \vec{s}}(x) d \vec{\eta} d \vec{s}\right|>\lambda / 4\right\}\right) \\
\lesssim & \lambda^{-2}\left\|\iint_{E^{l}} u^{\vec{\eta}, \vec{s}}(x) \sum_{n \geq 100} \sum_{j \in \mathbb{Z}} \sum_{v} G_{n, v}\left(I-P_{j-n \kappa}\right) T_{j}^{n, v}\left[a_{1}\right] \mathfrak{B}_{j-n}^{\vec{\eta}, \vec{s}}(x) d \vec{\eta} d \vec{s}\right\|_{2}^{2} \\
& +\lambda^{-1}\left\|\iint_{E^{l}} u^{\vec{\eta}, \vec{s}}(x) \sum_{n \geq 100} \sum_{j \in \mathbb{Z}} \sum_{v}\left(I-G_{n, v}\right)\left(I-P_{j-n \kappa}\right) T_{j}^{n, v}\left[a_{1}\right] \mathfrak{B}_{j-n}^{\vec{\eta}, \vec{s}}(x) d \vec{\eta} d \vec{s}\right\|_{1} .
\end{aligned}
$$


Using Lemma 2.5, we can get the desired estimate of the second term above. By Minkowski's inequality and Lemma 2.4 , the first term above is bounded by

$$
\begin{aligned}
& \lambda^{-2}\left(\sum_{n \geq 100}\left\|\iint_{E^{l}} u^{\vec{\eta}, \vec{s}}(x) \sum_{j \in \mathbb{Z}} \sum_{v} G_{n, v}\left(I-P_{j-n \kappa}\right) T_{j}^{n, v}\left[a_{1}\right] \mathfrak{B}_{j-n}^{\vec{\eta}, \vec{s}}(x) d \vec{\eta} d \vec{s}\right\|_{2}\right)^{2} \\
\lesssim & \lambda^{-2}\left(\sum_{n \geq 100}\left(2^{-n \gamma} \lambda\|f\|_{1}\right)^{\frac{1}{2}}\right)^{2} \lesssim \lambda^{-1}\|f\|_{1} .
\end{aligned}
$$

We hence complete the proof of Theorem 1.1 once Lemmas 2.2-2.5 hold.

\section{Proofs of Lemmas 2.2-2.4}

\subsection{Proof of Lemma 2.2}

By the definitions of $T_{j}\left[a_{1}\right]$ and $T_{j}^{n}\left[a_{1}\right]$, we have

$$
\begin{aligned}
\left\|T_{j}\left[a_{1}\right] f-T_{j}^{n}\left[a_{1}\right] f\right\|_{1} & =\int_{\mathbb{R}^{d}}\left|\int_{\mathbb{R}^{d}}\left(K_{j}(x-y) m_{x, y} a_{1}-K_{j}^{n}(x-y) m_{x, y}^{n} a_{1}\right) f(y) d y\right| d x \\
& \leq I+I I,
\end{aligned}
$$

where

$$
\begin{aligned}
& I=\int_{\mathbb{R}^{d}}\left|\int_{\mathbb{R}^{d}}\left(K_{j}(x-y)-K_{j}^{n}(x-y)\right) m_{x, y} a_{1} \cdot f(y) d y\right| d x, \\
& I I=\int_{\mathbb{R}^{d}}\left|\int_{\mathbb{R}^{d}} K_{j}^{n}(x-y)\left(m_{x, y} a_{1}-m_{x, y}^{n} a_{1}\right) f(y) d y\right| d x .
\end{aligned}
$$

Consider I firstly. By the definition of $K_{j}^{n}(x)$, we have

$$
K_{j}(x-y)-K_{j}^{n}(x-y)=\int \eta_{j-l_{\tau}(n)}(z)\left(K_{j}(x-y)-K_{j}(x-y-z)\right) d z .
$$

Notice that

$$
\begin{aligned}
& \left|K_{j}(x-y)-K_{j}(x-y-z)\right| \\
\leq & \left|\phi_{j}(x-y)(K(x-y)-K(x-y-z))\right|+\left|\phi_{j}(x-y)-\phi_{j}(x-y-z)\right||K(x-y-z)| \\
:= & A+B .
\end{aligned}
$$

Consider the first term $A$. Note that $|z| \leq 2^{j-l_{\tau}(n)}$ and $2^{j-1} \leq|x-y| \leq 2^{j+1}$, then we have $2|z|<|x-y|$. By the regularity condition (1.1b), we have

$$
A \lesssim \frac{|z|^{\delta}}{|x-y|^{d+\delta}} \chi_{\left\{2^{j-1} \leq|x-y| \leq 2^{j+1}\right\}} \lesssim 2^{-\tau n} 2^{-j d} \chi_{\left\{2^{j-1} \leq|x-y| \leq 2^{j+1}\right\}} .
$$


For the second therm $B$, by the fact $|z| \leq 2^{j-l_{\tau}(n)}$ and the support of $\phi_{j}$, we have $|x-y| \approx$ $|x-z-y|$ and $2^{j-2} \leq|x-y| \leq 2^{j+2}$. By (1.1a), we get

$$
B \lesssim \frac{2^{-j}|z|}{|x-z-y|^{d}} \chi_{\left\{2^{j-2} \leq|x-y| \leq 2^{j+2}\right\}} \lesssim 2^{-\tau n} 2^{-j d} \chi_{\left\{2^{j-2} \leq|x-y| \leq 2^{j+2}\right\}}
$$

As for $I I$, we have

$$
\left|m_{x, y} a_{1}-m_{x, y}^{n} a_{1}\right|=\left|\int_{0}^{1}\left(1-\rho_{n}(s)\right) a_{1}(s x+(1-s) y) d s\right| \lesssim 2^{-\tau n}\left\|a_{1}\right\|_{\infty} .
$$

Combining the above three estimates and applying Minkowski's inequality, we have

$$
\begin{aligned}
& \left\|T_{j}\left[a_{1}\right] f-T_{j}^{n}\left[a_{1}\right] f\right\|_{1} \\
\lesssim & 2^{-\tau n}\left\|a_{1}\right\|_{\infty} \int_{\mathbb{R}^{d}} \int_{2^{j-2} \leq|x-y| \leq 2^{j+2}} 2^{-j d} \int_{\mathbb{R}^{d}} \eta_{j-l_{\tau}(n)}(z) d z|f(y)| d y d x \\
\lesssim & 2^{-\tau n}\left\|a_{1}\right\|_{\infty} 2^{-j d} \int_{\mathbb{R}^{d}} \int_{2^{j-2} \leq|x-y| \leq 2^{j+2}}|f(y)| d y d x \\
\lesssim & 2^{-\tau n}\left\|a_{1}\right\|_{\infty}\|f\|_{1} .
\end{aligned}
$$

By Chebyshev's inequality, Minkowski's inequality, the estimates above and the property (iv) in Lemma 2.1, we get the bound

$$
\begin{aligned}
& m\left(\left\{x \in \mathbb{R}^{d}:\left|\iint_{E^{l}} u^{\vec{\eta}, \vec{s}}(x) \sum_{n \geq 100} \sum_{j \in \mathbb{Z}}\left(T_{j}\left[a_{1}\right] \mathfrak{B}_{j-n}^{\vec{\eta}, \vec{s}}(x)-T_{j}^{n}\left[a_{1}\right] \mathfrak{B}_{j-n}^{\vec{\eta}, \vec{s}}(x)\right) d \vec{\eta} d \vec{s}\right|>\lambda / 2\right\}\right) \\
\lesssim & \lambda^{-1} \sum_{n \geq 100} \iint_{E^{l}}\left|\prod_{i=2}^{l} \hat{a}_{i}\left(\eta_{i}\right)\right| \sum_{j}\left\|T_{j}\left[a_{1}\right] \mathfrak{B}_{j-n}^{\vec{\eta}, \vec{s}}-T_{j}^{n}\left[a_{1}\right] \mathfrak{B}_{j-n}^{\vec{\eta}, \vec{s}}\right\|_{1} d \vec{\eta} d \vec{s} \\
\lesssim & \lambda^{-1}\left\|a_{1}\right\|_{\infty}\left(\prod_{i=2}^{l}\left\|\hat{a}_{i}\right\|_{1}\right) \sum_{n \geq 100} 2^{-\tau n}\|f\|_{1} \lesssim \lambda^{-1}\|f\|_{1},
\end{aligned}
$$

which is the required estimate.

\subsection{Proof of Lemma 2.3}

Since $\mathfrak{B}_{j-n}^{\vec{\eta}, \vec{s}}=\sum_{l(Q)=2^{j-n}} b_{Q}^{\vec{\eta}, \vec{s}}$, we only need to consider a fixed $b_{Q}^{\vec{\eta}, \vec{s}}$ with $l(Q)=2^{j-n}$. By applying Fubini's theorem, one may write

$$
\begin{aligned}
& P_{j-n \kappa} T_{j}^{n}\left[a_{1}\right] b_{Q}^{\vec{\eta}, \vec{s}}(x) \\
= & \int_{0}^{1} \rho_{n}(s) \int_{\mathbb{R}^{d}} b_{Q}^{\vec{\eta}, \vec{s}}(y) \int_{\mathbb{R}^{d}} \eta_{j-n \kappa}(x-w) K_{j}^{n}(w-y) a_{1}(s w+(1-s) y) d w d y d s .
\end{aligned}
$$


Making a change of variables $z=w+\frac{1-s}{s} y$, one may get

$$
\begin{aligned}
& P_{j-n \kappa} T_{j}^{n}\left[a_{1}\right] b_{Q}^{\vec{\eta}, \vec{s}}(x) \\
= & \int_{0}^{1} \rho_{n}(s) \int_{\mathbb{R}^{d}} a_{1}(s z) \int_{\mathbb{R}^{d}} \eta_{j-n \kappa}\left(x-z+\frac{1-s}{s} y\right) K_{j}^{n}\left(z-\frac{y}{s}\right) b_{Q}^{\vec{\eta}, \vec{s}}(y) d y d z d s .
\end{aligned}
$$

By using the cancellation of $b_{Q}^{\vec{\eta}, \vec{s}}$ (see the property of (iv) in Lemma 2.1), one may write

$$
\begin{aligned}
& \left|P_{j-n \kappa} T_{j}^{n}\left[a_{1}\right] b_{Q}^{\vec{\eta}, \vec{s}}(x)\right| \\
= & \mid \int_{0}^{1} \rho_{n}(s) \int_{\mathbb{R}^{d}} a_{1}(s z) \int_{\mathbb{R}^{d}} b_{Q}^{\vec{\eta}, \vec{s}}(y) \\
& \quad \times\left(\eta_{j-n \kappa}\left(x-z+\frac{1-s}{s} y\right) K_{j}^{n}\left(z-\frac{y}{s}\right)-\eta_{j-n \kappa}\left(x-z+\frac{1-s}{s} y_{0}\right) K_{j}^{n}\left(z-\frac{y_{0}}{s}\right)\right) d y d z d s \mid \\
\leq & I+I I,
\end{aligned}
$$

where $y_{0}$ is the center of $Q$ and

$$
\begin{aligned}
I=\mid & \int_{0}^{1} \rho_{n}(s) \int_{\mathbb{R}^{d}}\left|a_{1}(s z)\right| \int_{\mathbb{R}^{d}}\left|b_{Q}^{\vec{\eta}, \vec{s}}(y)\right| \\
& \times\left|\eta_{j-n \kappa}\left(x-z+\frac{1-s}{s} y\right)-\eta_{j-n \kappa}\left(x-z+\frac{1-s}{s} y_{0}\right)\right|\left|K_{j}^{n}\left(z-\frac{y}{s}\right)\right| d y d z d s
\end{aligned}
$$

and

$$
\begin{aligned}
I I=\mid & \int_{0}^{1} \rho_{n}(s) \int_{\mathbb{R}^{d}}\left|a_{1}(s z)\right| \int_{\mathbb{R}^{d}}\left|b_{Q}^{\vec{\eta}, \vec{s}}(y)\right| \\
& \times\left|\eta_{j-n \kappa}\left(x-z+\frac{1-s}{s} y_{0}\right)\right|\left|K_{j}^{n}\left(z-\frac{y}{s}\right)-K_{j}^{n}\left(z-\frac{y_{0}}{s}\right)\right| d y d z d s .
\end{aligned}
$$

Since $l(Q)=2^{j-n}$, we have $\left|y-y_{0}\right| \lesssim 2^{j-n}$. Using the mean value formula, one may have

$$
\begin{aligned}
I \lesssim & \left\|a_{1}\right\|_{\infty} \int_{0}^{1} \rho_{n}(s) \int_{\mathbb{R}^{d}} \int_{\mathbb{R}^{d}}\left|b_{Q}^{\vec{\eta}, \vec{s}}(y)\right| \frac{1-s}{s} 2^{-j+\kappa n}\left|y-y_{0}\right|\left|K_{j}^{n}\left(z-\frac{y}{s}\right)\right| d y d z d s \\
& \lesssim 2^{(-1+\kappa) n}\left\|a_{1}\right\|_{\infty} \int_{0}^{1} \rho_{n}(s) s^{-1} d s\left\|K_{j}^{n}\right\|_{1}\left\|b_{Q}^{\vec{\eta}, \vec{s}}\right\|_{1} \lesssim n 2^{(-1+\kappa) n}\left\|b_{Q}^{\vec{\eta}, \vec{s}}\right\|_{1} .
\end{aligned}
$$

Similarly, by the mean value formula and (2.8), one may get

$$
\begin{aligned}
I I & \lesssim\left\|a_{1}\right\|_{\infty} \int_{0}^{1} \rho_{n}(s) \int_{\mathbb{R}^{d}} \int_{\mathbb{R}^{d}}\left|b_{Q}^{\vec{\eta}, \vec{s}}(y)\right| s^{-1}\left|y-y_{0}\right| \int_{0}^{1}\left|\nabla K_{j}^{n}\left(z-\frac{t y+(1-t) y_{0}}{s}\right)\right| d t d y d z d s \\
& \lesssim 2^{j-n}\left\|a_{1}\right\|_{\infty} \int_{0}^{1} \rho_{n}(s) s^{-1} d s\left\|\nabla K_{j}^{n}\right\|_{1}\left\|b_{Q}^{\vec{\eta}, \vec{s}}\right\|_{1} \lesssim n 2^{\left(-1+\tau \delta^{-1}\right) n}\left\|b_{Q}^{\vec{\eta}, \vec{s}}\right\|_{1} .
\end{aligned}
$$

Combining the estimates of $I$ and $I I$ and summing over all $Q$ with $l(Q)=2^{j-n}$, we finish the proof of Lemma 2.3. 


\subsection{Proof of Lemma 2.4}

The proof of this lemma is quite similar to that of Proposition 2.4 in [18]. As usually, we adopt the $T T^{*}$ method in the $L^{2}$ estimate. We also use some orthogonality argument based on the following observation of the support of $\mathcal{F}\left(G_{n, v}\left(I-P_{j-n \kappa}\right) T_{j}^{n, v}\left[a_{1}\right]\right)$ : For a fixed $n \geq 100$, we have

$$
\sup _{\xi \neq 0} \sum_{v}\left|\Phi^{2}\left(2^{n \gamma}\left\langle e_{v}^{n}, \xi /|\xi|\right\rangle\right)\right| \lesssim 2^{n \gamma(d-2)}
$$

In fact, by the homogeneity of $\Phi^{2}\left(2^{n \gamma}\left\langle e_{v}^{n}, \xi /|\xi|\right\rangle\right)$, it suffices to take the supremum over the surface $S^{d-1}$. For $|\xi|=1$ and $\xi \in \operatorname{supp} \Phi\left(2^{n \gamma}\left\langle e_{v}^{n}, \xi /|\xi|\right\rangle\right)$, denote by $\xi^{\perp}$ the hyperplane perpendicular to $\xi$. Then

$$
\operatorname{dist}\left(e_{v}^{n}, \xi^{\perp}\right) \lesssim 2^{-n \gamma} .
$$

Since the mutual distance of $e_{v}^{n \text { 's }}$ is bounded by $2^{-n \gamma-4}$, there are at most $2^{n \gamma(d-2)}$ vectors satisfy (3.3). We hence get (3.2).

By applying Plancherel's theorem and Cauchy-Schwarz inequality, we have

$$
\begin{aligned}
&\left\|\iint_{E^{l}} u^{\vec{\eta}, \vec{s}}(x) \sum_{j \in \mathbb{Z}} \sum_{v} G_{n, v}\left(I-P_{j-n \kappa}\right) T_{j}^{n, v}\left[a_{1}\right] \mathfrak{B}_{j-n}^{\vec{\eta}, \vec{s}}(x) d \vec{\eta} d \vec{s}\right\|_{2}^{2} \\
&=\left(\iint_{E^{l}}\left|\prod_{i=2}^{l} \hat{a}_{i}\left(\eta_{i}\right)\right|\left\|\sum_{v} \Phi\left(2^{n \gamma}\left\langle e_{v}^{n}, \xi /|\xi|\right\rangle\right) \mathcal{F}\left(\sum_{j}\left(I-P_{j-n \kappa}\right) T_{j}^{n, v}\left[a_{1}\right] \mathfrak{B}_{j-n}^{\vec{\eta}, \vec{s}}\right)(\xi)\right\|_{2} d \vec{\eta} d \vec{s}\right)^{2} \\
& \lesssim 2^{n \gamma(d-2)}\left(\iint_{E^{l}}\left|\prod_{i=2}^{l} \hat{a}_{i}\left(\eta_{i}\right)\right|\left\|\sum_{v}\left|\mathcal{F}\left(\sum_{j}\left(I-P_{j-n \kappa}\right) T_{j}^{n, v}\left[a_{1}\right] \mathfrak{B}_{j-n}^{\vec{\eta}, \vec{s}}\right)\right|^{2}\right\|_{1}^{\frac{1}{2}} d \vec{\eta} d \vec{s}\right)^{2} \\
& \lesssim 2^{n \gamma(d-2)}\left(\iint_{E^{l}}\left|\prod_{i=2}^{l} \hat{a}_{i}\left(\eta_{i}\right)\right|\left(\sum_{v}\left\|\sum_{j}\left(I-P_{j-n \kappa}\right) T_{j}^{n, v}\left[a_{1}\right] \mathfrak{B}_{j-n}^{\vec{\eta}, \vec{s}}\right\|_{2}^{2}\right)^{\frac{1}{2}} d \vec{\eta} d \vec{s}\right)^{2} .
\end{aligned}
$$

Once it is showed that for a fixed $e_{v}^{n}, \vec{\eta}, \vec{s}$,

$$
\left\|\sum_{j}\left(I-P_{j-n \kappa}\right) T_{j}^{n, v}\left[a_{1}\right] \mathfrak{B}_{j-n}^{\vec{\eta}, \vec{s}}\right\|_{2}^{2} \lesssim 2^{-2 n \gamma(d-1)} \lambda\|f\|_{1},
$$

then by $\operatorname{card}\left(\Theta_{n}\right) \lesssim 2^{n \gamma(d-1)}$, and apply (3.4) and (3.5) we get

$$
\left\|\iint_{E^{l}} u^{\vec{\eta}, \vec{s}}(x) \sum_{j \in \mathbb{Z}} \sum_{v} G_{n, v}\left(I-P_{j-n \kappa}\right) T_{j}^{n, v}\left[a_{1}\right] \mathfrak{B}_{j-n}^{\vec{\eta}, \vec{s}}(x) d \vec{\eta} d \vec{s}\right\|_{2}^{2} \lesssim 2^{-n \gamma} \lambda\|f\|_{1},
$$

which is just the desired bound of Lemma 2.4. Thus, to finish the proof of Lemma 2.4, it is enough to prove (3.5). By applying (2.7), the support of $\Gamma_{v}^{n}$ and $0<\gamma<\kappa<1$, we have

$$
\left|\left(I-P_{j-n \kappa}\right) T_{j}^{n, v}\left[a_{1}\right] \mathfrak{B}_{j-n}^{\vec{\eta}, \vec{s}}(x)\right| \lesssim H_{j}^{n, v} *\left|\mathfrak{B}_{j-n}^{\vec{\eta}, \vec{s}}\right|(x),
$$


where $H_{j}^{n, v}(x):=2^{-j d} \chi_{E_{j}^{n, v}}(x)$ and $\chi_{E_{j}^{n, v}}(x)$ is a characteristic function of the set

$$
E_{j}^{n, v}:=\left\{x \in \mathbb{R}^{d}:\left|\left\langle x, e_{v}^{n}\right\rangle\right| \leq 2^{j+2},\left|x-\left\langle x, e_{v}^{n}\right\rangle e_{v}^{n}\right| \leq 2^{j+2-n \gamma}\right\}
$$

For a fixed $e_{v}^{n}$, we write

$$
\begin{aligned}
\left\|\sum_{j} T_{j}^{n, v}\left[a_{1}\right] \mathfrak{B}_{j-n}^{\vec{\eta}, \vec{s}}\right\|_{2}^{2} \lesssim & \sum_{j} \int_{\mathbb{R}^{d}} H_{j}^{n, v} * H_{j}^{n, v} *\left|\mathfrak{B}_{j-n}^{\vec{\eta}, \vec{s}}\right|(x) \cdot\left|\mathfrak{B}_{j-n}^{\vec{\eta}, \vec{s}}(x)\right| d x \\
& +\sum_{j} \sum_{i=-\infty}^{j-1} \int_{\mathbb{R}^{d}} H_{j}^{n, v} * H_{i}^{n, v} *\left|\mathfrak{B}_{i-n}^{\vec{\eta}, \vec{s}}\right|(x) \cdot\left|\mathfrak{B}_{j-n}^{\vec{\eta}, \vec{s}}(x)\right| d x .
\end{aligned}
$$

Observe that $\left\|H_{i}^{n, v}\right\|_{1} \lesssim 2^{-i d} m\left(E_{i}^{n, v}\right) \lesssim 2^{-n \gamma(d-1)}$, therefore for any $i \leq j$,

$$
H_{j}^{n, v} * H_{i}^{n, v}(x) \leq 2^{-n \gamma(d-1)} 2^{-j d} \chi_{\widetilde{E}_{j}^{n, v}}
$$

where $\widetilde{E}_{j}^{n, v}=E_{j}^{n, v}+E_{j}^{n, v}$. Hence for a fixed $\vec{\eta}, \vec{s}, j, n, e_{v}^{n}$ and $x$, we have

$$
\begin{aligned}
& H_{j}^{n, v} * H_{j}^{n, v} *\left|\mathfrak{B}_{j-n}^{\vec{\eta}, \vec{s}}\right|(x)+\sum_{i=-\infty}^{j-1} H_{j}^{n, v} * H_{i}^{n, v} *\left|\mathfrak{B}_{i-n}^{\vec{\eta}, \vec{s}}\right|(x) \\
\lesssim & 2^{-n \gamma(d-1)} 2^{-j d} \sum_{i \leq j} \int_{x+\widetilde{E}_{j}^{n, v}}\left|\mathfrak{B}_{i-n}^{\vec{\eta}, \vec{s}}(y)\right| d y \\
\lesssim & 2^{-n \gamma(d-1)} 2^{-j d} \sum_{i \leq j} \sum_{\substack{Q \in \mathfrak{D}_{i-n} \\
Q \cap\left\{x+\mathbb{E}_{j}^{n, v}\right\} \neq \varnothing}} \int_{\mathbb{R}^{d}}\left|b_{Q}^{\vec{\eta}, \vec{s}}(y)\right| d y \\
\lesssim & 2^{-n \gamma(d-1)} 2^{-j d} \sum_{i \leq j} \sum_{\substack{Q \in \mathfrak{D}_{i-n} \\
Q \cap\left\{x+\mathbb{E}_{j}^{n, v}\right\} \neq \varnothing}} \lambda|Q| \\
\lesssim & \lambda 2^{-2 n \gamma(d-1)},
\end{aligned}
$$

where in third inequality above, we use $\int\left|b_{Q}^{\vec{\eta}, \vec{s}}(y)\right| d y \lesssim \lambda|Q|$ (see the property (iv) in Lemma 2.1) and in the fourth inequality we use fact that the cubes in $Q$ are disjoint (see the property (i) in Lemma 2.1). By (3.6), (3.7) and the property (iv) in Lemma 2.1, we obtain

$$
\left\|\sum_{j}\left(I-P_{j-n \kappa}\right) T_{j}^{n, v}\left[a_{1}\right] \mathfrak{B}_{j-n}^{\vec{\eta}, \vec{s}}\right\|_{2}^{2} \lesssim \lambda 2^{-2 n \gamma(d-1)} \sum_{j}\left\|\mathfrak{B}_{j-n}^{\vec{\eta}, \vec{s}}\right\|_{1} \lesssim \lambda 2^{-2 n \gamma(d-1)}\|f\|_{1} .
$$

Hence, we complete the proof of Lemma 2.4. 


\section{Proof of Lemma 2.5}

To prove Lemma 2.5, we have to deal with some oscillatory integrals which come from $\left(I-G_{n, v}\right) T_{j}^{n, v}\left[a_{1}\right]$.

Before stating the proof of Lemma 2.5, let us give some notations. We first introduce the Littlewood-Paley decomposition. Let $\psi$ be a radial $C^{\infty}$ function such that $\psi(\xi)=1$ for $|\xi| \leq 1, \psi(\xi)=0$ for $|\xi| \geq 2$ and $0 \leq \psi(\xi) \leq 1$ for all $\xi \in \mathbb{R}^{d}$. Define $\beta_{k}(\xi)=\psi\left(2^{k} \xi\right)-\psi\left(2^{k+1} \xi\right)$, then $\beta_{k}$ is supported in $\left\{\xi: 2^{-k-1} \leq|\xi| \leq 2^{-k+1}\right\}$ and $\sum_{k} \beta_{k}(\xi)=1$ for $\xi \in \mathbb{R}^{d} \backslash\{0\}$. Choose $\tilde{\beta}$ be a radial $C^{\infty}$ function such that $\tilde{\beta}(\tilde{\xi})=1$ for $\frac{1}{2} \leq|\xi| \leq 2, \tilde{\beta}$ is supported in $\left\{\xi: \frac{1}{4} \leq|\xi| \leq 4\right\}$ and $0 \leq \tilde{\beta}(\xi) \leq 1$ for all $\xi \in \mathbb{R}^{d}$. Set $\tilde{\beta}_{k}(\tilde{\xi})=\tilde{\beta}\left(2^{k} \xi\right)$, then it is easy to see $\beta_{k}=\tilde{\beta}_{k} \beta_{k}$. Define the convolution operators $\Lambda_{k}$ and $\tilde{\Lambda}_{k}$ with Fourier multipliers $\beta_{k}$ and $\tilde{\beta}_{k}$, respectively. That is,

$$
\widehat{\Lambda_{k} f}(\xi)=\beta_{k}(\xi) \hat{f}(\xi), \quad \widehat{\tilde{\Lambda}_{k} f}(\xi)=\tilde{\beta}_{k}(\xi) \hat{f}(\xi) .
$$

Then by the construction of $\beta_{k}$ and $\tilde{\beta}_{k}$, we have

$$
\Lambda_{k}=\tilde{\Lambda}_{k} \Lambda_{k}, \quad I=\sum_{k \in \mathbb{Z}} \Lambda_{k}
$$

where $I$ is the identity. Write

$$
\left(I-G_{n, v}\right) T_{j}^{n, v}\left[a_{1}\right]=\sum_{k}\left(I-G_{n, v}\right) \Lambda_{k} T_{j}^{n, v}\left[a_{1}\right] .
$$

By using Minkowski's inequality,

$$
\begin{aligned}
& \left\|\iint_{E^{l}} u^{\vec{\eta}, \vec{s}}(x) \sum_{n \geq 100} \sum_{v} \sum_{j}\left(I-P_{j-n \kappa}\right)\left(I-G_{n, v}\right) T_{j}^{n, v}\left[a_{1}\right] \mathfrak{B}_{j-n}^{\vec{\eta}, \vec{s}}(x) d \vec{\eta} d \vec{s}\right\|_{1} \\
\leq & \sum_{n \geq 100} \sum_{v} \sum_{j} \sum_{k} \sum_{l(Q)=2^{j-n}} \iint_{E^{l}}\left|\prod_{i=2}^{l} \hat{a}_{i}\left(\eta_{i}\right)\right| \cdot\left\|\left(I-P_{j-n \kappa}\right)\left(I-G_{n, v}\right) \Lambda_{k} T_{j}^{n, v}\left[a_{1}\right] b_{Q}^{\vec{\eta}, \vec{s}}\right\|_{1} d \vec{\eta} d \vec{s} .
\end{aligned}
$$

Lemma 4.1. For a fixed $b_{Q}^{\vec{\eta}, \vec{s}}$ with $l(Q)=2^{j-n}$, there exists $N>0$, such that

$$
\left\|\left(I-P_{j-n \kappa}\right)\left(I-G_{n, v}\right) \Lambda_{k} T_{j}^{n, v}\left[a_{1}\right] b_{Q}^{\vec{\eta}, \vec{s}}\right\|_{1} \lesssim 2^{\delta^{-1} \tau n-n \gamma(d-1)+(-j+k)+n \gamma(1+2 N)}\left\|b_{Q}^{\vec{\eta}, \vec{s}}\right\|_{1} .
$$

Proof. First write

$$
\begin{aligned}
& \left\|\left(I-P_{j-n \kappa}\right)\left(I-G_{n, v}\right) \Lambda_{k} T_{j}^{n, v}\left[a_{1}\right] b_{Q}^{\vec{\eta}, \vec{s}}\right\|_{1} \\
= & \left\|\left(I-P_{j-n \kappa}\right) \tilde{\Lambda}_{k}\left(I-G_{n, v}\right) \Lambda_{k} T_{j}^{n, v}\left[a_{1}\right] b_{Q}^{\vec{\eta}, \vec{s}}\right\|_{1} \\
\leq & \left\|\left(I-P_{j-n \kappa}\right) \tilde{\Lambda}_{k}\right\|_{L^{1} \rightarrow L^{1}}\left\|\left(I-G_{n, v}\right) \Lambda_{k} T_{j}^{n, v}\left[a_{1}\right] b_{Q}^{\vec{\eta}, \vec{s}}\right\|_{1} .
\end{aligned}
$$


It is easy to see that $\left\|\left(I-P_{j-n \kappa}\right) \tilde{\Lambda}_{k}\right\|_{L^{1} \rightarrow L^{1}} \lesssim 1$ uniformly with $j, k, n \kappa$. Denote $h_{k, n, v}(\xi)=$ $\left(1-\Phi\left(2^{n \gamma}\left\langle e_{v}^{n}, \xi /|\xi|\right\rangle\right)\right) \beta_{k}(\xi)$. Applying Fubini's theorem, we may write

$$
\left(I-G_{n, v}\right) \Lambda_{k} T_{j}^{n, v}\left[a_{1}\right] b_{Q}^{\vec{\eta}, \vec{s}}(x)=: \int_{\mathbb{R}^{d}} D_{k}(x, y) b_{Q}^{\vec{\eta}, \vec{s}}(y) d y,
$$

where

$$
D_{k}(x, y)=\frac{1}{(2 \pi)^{d}} \int_{\mathbb{R}^{d}} e^{i x \cdot \xi} h_{k, n, v}(\xi) \int_{\mathbb{R}^{d}} e^{-i \xi^{\tau} \cdot \omega} K_{j}^{n, v}(\omega-y) m_{\omega, y}^{n} a_{1} d \omega d \xi .
$$

Next we make a change of variables to polar coordinate $\omega-y=r \theta$. By Fubini's theorem, $D_{k}(x, y)$ can be written as

$$
\frac{1}{(2 \pi)^{d}} \int_{\mathbb{S}^{d-1}} \Gamma_{v}^{n}(\theta)\left\{\int_{\mathbb{R}^{d}} \int_{0}^{\infty} e^{i\langle x-y-r \theta, \xi\rangle} h_{k, n, v}(\xi) K_{j}^{n}(r \theta) r^{d-1}\left(m_{y+r \theta, y}^{n} a_{1}\right) d r d \xi\right\} d \sigma(\theta) .
$$

By the support of $K_{j}^{n}(x)$ in (2.7), we have $2^{j-2} \leq r \leq 2^{j+2}$. Since $\theta \in \operatorname{supp} \Gamma_{v}^{n}$, then $\left|\theta-e_{v}^{n}\right| \leq$ $2^{-n \gamma}$. Using the support of $\Phi$, we see $\left|\left\langle e_{v}^{n}, \xi /|\xi|\right\rangle\right| \geq 2^{1-n r}$. Thus,

$$
|\langle\theta, \xi /|\xi|\rangle| \geq\left|\left\langle e_{v}^{n}, \xi /|\xi|\right\rangle\right|-\left|\left\langle e_{v}^{n}-\theta, \xi /|\xi|\right\rangle\right| \geq 2^{-n \gamma} .
$$

Integrating by parts with $r, D_{k}(x, y)$ can be rewritten as

$$
\frac{1}{(2 \pi)^{d}} \int_{S^{d-1}} \Gamma_{v}^{n}(\theta)\left\{\int_{\mathbb{R}^{d}} \int_{0}^{\infty} e^{i\langle x-y-r \theta, \xi\rangle} \frac{h_{k, n, v}(\xi)}{i\langle\theta, \xi\rangle} \partial_{r}\left[K_{j}^{n}(r \theta) r^{d-1} m_{y+r \theta, y}^{n} a_{1}\right] d r d \xi\right\} d \sigma(\theta) .
$$

After integrating by parts with $r$, integrating by parts with $\xi$, the integral $D_{k}(x, y)$ can be rewritten as

$$
\begin{aligned}
& \frac{1}{(2 \pi)^{d}} \int_{S^{d-1}} \Gamma_{v}^{n}(\theta) \int_{\mathbb{R}^{d}} e^{i\langle x-y-r \theta, \xi\rangle} \int_{0}^{\infty} \partial_{r}\left(K_{j}^{n}(r \theta) r^{d-1} m_{y+r \theta, y}^{n} a_{1}\right) \\
& \times \frac{\left(I-2^{-2 k} \Delta_{\xi}\right)^{N}}{\left(1+2^{-2 k}|x-y-r \theta|^{2}\right)^{N}}\left(h_{k, n, v}(\xi)(i\langle\theta, \xi\rangle)^{-1}\right) d r d \xi d \sigma(\theta) .
\end{aligned}
$$

In the following, we give an explicit estimate of the term in (4.6). By the definition of $K_{j}^{n}(x)$, we have

$$
\begin{aligned}
\left|\partial_{x}^{\alpha} K_{j}^{n}(x)\right| & =2^{-\left(j-l_{\tau}(n)\right)|\alpha|}\left|\int\left(\partial_{x}^{\alpha} \eta\right)_{j-l_{\tau}(n)}(x-z) K_{j}(z) d z\right| \\
& \leq 2^{-\left(j-l_{\tau}(n)\right)|\alpha|}\left\|K_{j}\right\|_{\infty}\left\|\partial_{x}^{\alpha} \eta\right\|_{1} \lesssim 2^{-\left(j-l_{\tau}(n)\right)|\alpha|-j d,},
\end{aligned}
$$

where the third inequality follows from (2.7). Observe

$$
\left|\partial_{r}\left(m_{y+r \theta, y}^{n} a_{1}\right)\right|=\left|\partial_{r}\left(\frac{1}{r} \int_{0}^{r} \rho_{n}\left(\frac{s}{r}\right) a_{1}(y+s \theta) d s\right)\right| \lesssim \frac{1}{r} 2^{l_{\tau}(n)}\left\|a_{1}\right\|_{\infty} .
$$


By using product rule, (4.7), (4.8) and $2^{j-2} \leq r \leq 2^{j+2}$,

$$
\left|\partial_{r}\left(K_{j}^{n}(r \theta) r^{d-1}\left(m_{y+r \theta, y}^{n} a_{1}\right)\right)\right| \lesssim 2^{l_{\tau}(n)-2 j}\left\|a_{1}\right\|_{\infty} .
$$

By (4.5), we have

$$
\left|(-i\langle\theta, \xi\rangle)^{-1} \cdot h_{k, n, v}(\xi)\right| \lesssim|\langle\theta, \xi\rangle|^{-1} \lesssim 2^{n \gamma+k}
$$

Now using product rule,

$$
\left|\partial_{\xi_{i}} h_{k, n, v}(\xi)\right|=\left|-\partial_{\xi_{i}}\left[\Phi\left(2^{n \gamma}\left\langle e_{v}^{n}, \xi /|\xi|\right\rangle\right)\right] \cdot \beta_{k}(\xi)+\partial_{\xi_{i}} \beta_{k}(\xi) \cdot\left(1-\Phi\left(2^{n \gamma}\left\langle e_{v}^{n}, \xi /|\xi|\right\rangle\right)\right)\right| \lesssim 2^{n \gamma+k} .
$$

Therefore by induction, we have $\left|\partial_{\xi}^{\alpha} h_{k, n, v}(\xi)\right| \lesssim 2^{(n \gamma+k)|\alpha|}$ for any multi-indices $\alpha \in \mathbb{Z}_{+}^{n}$. By using product rule again and (4.5), we have

$$
\begin{aligned}
\left.\mid \partial_{\xi_{i}}^{2}(\langle\theta, \xi\rangle)^{-1} h_{k, n, v}(\xi)\right) \mid & =\left|\langle\theta, \xi\rangle^{-3} \cdot 2 \theta_{i}^{2} \cdot h_{k, n, v}-2\langle\theta, \xi\rangle^{-2} \cdot \theta_{i} \partial_{\xi_{i}} h_{k, n, v}(\xi)+\langle\theta, \xi\rangle^{-1} \partial_{\xi_{i}}^{2} h_{k, n, v}(\xi)\right| \\
& \lesssim 2^{3(n \gamma+k)} .
\end{aligned}
$$

This implies the follow inequality

$$
2^{-2 k}\left|\Delta_{\xi}\left[(\langle\theta, \xi\rangle)^{-1} h_{k, n, v}(\xi)\right]\right| \lesssim 2^{(n \gamma+k)+2 n \gamma} .
$$

Proceeding by induction, we have

$$
\left|\left(I-2^{-2 k} \Delta_{\xi}\right)^{N}\left[\langle\theta, \xi\rangle^{-1} h_{k, n, v}(\xi)\right]\right| \lesssim 2^{(n \gamma+k)+2 n \gamma N} .
$$

Now we choose $N=\left[\frac{d}{2}\right]+1$. By (4.3) and Minkowski's inequality,

$$
\left\|\left(I-G_{n, v}\right) \Lambda_{k} T_{j}^{n, v}\left[a_{1}\right] b_{Q}^{\vec{\eta}, \vec{s}}\right\|_{1} \leq \int\left\|D_{k}(\cdot, y)\right\|_{1}\left|b_{Q}^{\vec{\eta}, \vec{s}}(y)\right| d y .
$$

So we need to get the $L^{1}$ estimate of (4.4), by the support of $h_{k, n, v}$,

$$
\int_{\operatorname{supp}\left(h_{k, n, v}\right)} \int\left(1+2^{-2 k}|x-y-r \theta|^{2}\right)^{-N} d x d \xi \leq C .
$$

Integrating with $r$, we get a bound $2^{j}$. Then integrating with $\theta$, we get a bound $2^{-n \gamma(d-1)}$. Combining (4.9), (4.10) and the above estimates,

$$
\left\|D_{k}(\cdot, y)\right\|_{1} \lesssim 2^{\delta^{-1} \tau n-n \gamma(d-1)+(-j+k)+n \gamma(1+2 N)} .
$$

Hence we complete the proof of Lemma 4.1 with $N=\left[\frac{d}{2}\right]+1$.

Lemma 4.2. For a fixed $b_{Q}^{\vec{\eta}, \vec{s}}$ with $l(Q)=2^{j-n}$, then

$$
\left\|\left(I-P_{j-n \kappa}\right)\left(I-G_{n, v}\right) \Lambda_{k} T_{j}^{n, v}\left[a_{1}\right] b_{Q}^{\vec{\eta}, \vec{s}}\right\|_{1} \lesssim 2^{-n \gamma(d-1)+j-n \kappa-k}\left\|b_{Q}^{\vec{\eta}, \vec{s}}\right\|_{1} .
$$


Proof. We write

$$
\begin{aligned}
& \left\|\left(I-P_{j-n \kappa}\right)\left(I-G_{n, v}\right) \Lambda_{k} T_{j}^{n, v}\left[a_{1}\right] b_{Q}^{\vec{\eta}, \vec{s}}\right\|_{1} \\
= & \left\|\left(I-P_{j-n \kappa}\right) \tilde{\Lambda}_{k}\left(I-G_{n, v}\right) \Lambda_{k} T_{j}^{n, v}\left[a_{1}\right] b_{Q}^{\vec{\eta}, \vec{s}}\right\|_{1} \\
\leq & \left\|\left(I-P_{j-n \kappa}\right) \tilde{\Lambda}_{k}\right\|_{L^{1} \rightarrow L^{1}}\left\|\left(I-G_{n, v}\right) \Lambda_{k}\right\|_{L^{1} \rightarrow L^{1}}\left\|T_{j}^{n, v}\left[a_{1}\right] b_{Q}^{\vec{\eta}, \vec{s}}\right\|_{1} .
\end{aligned}
$$

By using Minkowski's inequality, one can easily get

$$
\left\|T_{j}^{n, v}\left[a_{1}\right] b_{Q}^{\vec{r}, \vec{s}}\right\|_{1} \lesssim 2^{-n \gamma(d-1)}\left\|b_{Q}^{\vec{\eta}, \vec{s}}\right\|_{1}
$$

Now we claim that

$$
\begin{aligned}
& \left\|\left(I-P_{j-n \kappa}\right) \tilde{\Lambda}_{k}\right\|_{L^{1} \rightarrow L^{1}} \lesssim 2^{j-n \kappa-k}, \\
& \left\|\left(I-G_{n, v}\right) \Lambda_{k}\right\|_{L^{1} \rightarrow L^{1}} \lesssim 1 .
\end{aligned}
$$

Combining (4.11), (4.12a) and (4.12b), one get the asserted bound. So to finish the proof, it suffice to show (4.12a) and (4.12b). Write

$$
\begin{aligned}
\left(I-P_{j-n \kappa}\right) \tilde{\Lambda}_{k} f(x) & =\widehat{\tilde{\beta}}_{k} * f(x)-\eta_{j-n \kappa} * \widehat{\tilde{\beta}}_{k} f(x) \\
& =\int_{\mathbb{R}^{d}} \int_{\mathbb{R}^{d}}\left(\widehat{\tilde{\beta}}_{k}(x-y)-\widehat{\widetilde{\beta}}_{k}(x-y-z)\right) \eta_{j-n \kappa}(z) d z \cdot f(y) d y .
\end{aligned}
$$

By using the mean value formula, one may get

$$
\widehat{\widetilde{\beta}_{k}}(x-y)-\widehat{\widetilde{\beta}}_{k}(x-y-z)=\int_{0}^{1}\left\langle z, \nabla \widehat{\widetilde{\beta}_{k}}(x-y-s z)\right\rangle d s .
$$

Utilize the Fubini's theorem, one may get

$$
\left\|\left(I-P_{j-n \kappa}\right) \tilde{\Lambda}_{k} f\right\|_{1} \lesssim 2^{j-n \kappa-k}\|\nabla \widetilde{\tilde{\beta}}\|_{1}\|\eta\|_{1}\|f\|_{1} \lesssim 2^{j-n \kappa-k}\|f\|_{1} .
$$

Thus we prove (4.12a). To prove (4.12b), it is enough to show that the function $h_{k, n, v}(\xi)=$ $\left(1-\Phi\left(2^{n \gamma}\left\langle e_{v}^{n}, \xi /|\xi|\right\rangle\right)\right) \beta_{k}(\xi)$ is a $L^{1}$ Fourier multiplier. Let $A_{k}^{n, v}$ be an invertible transform with $A_{k}^{n, v} e^{n, v}=2^{-n \gamma-k} e^{n, v}$ and $A_{k}^{n, v} y=2^{-k} y$ if $\left\langle y, e^{n, v}\right\rangle=0$. For all $\alpha \in \mathbb{Z}_{+}^{d}$, it is straightforward to check that

$$
\left\|\partial^{\alpha}\left(h_{k, n, v}\left(A_{k}^{n, v} \cdot\right)\right)\right\|_{2} \lesssim 1
$$

uniformly with $k, n, v$. By the Berenstein multiplier theorem (e.g., see Lemma 6.1 .5 in [1]),

$$
\left\|\left(I-G_{n, v}\right) \Lambda_{k}\right\|_{L^{1} \rightarrow L^{1}} \lesssim\left\|h_{k, n, v}\left(A_{k}^{n, v} \cdot\right)\right\|_{2}^{\frac{1}{2}} \sum_{|\alpha|=n}\left\|\partial^{\alpha}\left(h_{k, n, v}\left(A_{k}^{n, v} \cdot\right)\right)\right\|_{2}^{\frac{1}{2}} \lesssim 1,
$$

which completes the proof of $(4.12 b)$. 
Proof of Lemma 2.5. Let $\varepsilon_{0}$ satisfy $0<\varepsilon_{0}<1$ and will be chosen later. By (4.1), Lemma 4.1, Lemma 4.2 card $\Theta_{n} \lesssim 2^{n \gamma(d-1)}$, the property (iv) in Lemma 2.1 and the fact $\left[n \varepsilon_{0}\right] \leq n \varepsilon_{0}<$ $\left[n \varepsilon_{0}\right]+1$, one obtain

$$
\begin{aligned}
& \left\|\iint_{E^{l}} u^{\vec{\eta}, \vec{s}}(x) \sum_{n \geq 100} \sum_{v} \sum_{j}\left(I-P_{j-n \kappa}\right)\left(I-G_{n, v}\right) T_{j}^{n, v}\left[a_{1}\right] \mathfrak{B}_{j-n}^{\vec{\eta}, \vec{s}}(x) d \eta d s\right\|_{1} \\
\leq & \left(\sum_{n \geq 100} \sum_{v} \sum_{j} \sum_{k<j-\left[n \varepsilon_{0}\right]} \sum_{l(Q)=2^{j-n}}+\sum_{n \geq 100} \sum_{v} \sum_{j} \sum_{k \geq j-\left[n \varepsilon_{0}\right] l(Q)=2^{j-n}} \sum_{j}\right) \\
& \iint_{E^{l}}\left|\prod_{i=2}^{l} \hat{a}_{i}\left(\eta_{i}\right)\right| \cdot\left\|\left(I-P_{j-n \kappa}\right)\left(I-G_{n, v}\right) \Lambda_{k} T_{j}^{n, v}\left[a_{1}\right] b_{Q}^{\vec{\eta}, \vec{s}}\right\|_{1} d \eta d s \\
\lesssim & \sum_{n \geq 100}\left(2^{s_{1} n}+2^{s_{2} n}\right) \iint_{E^{l}}\left|\prod_{i=2}^{l} \hat{a}_{i}\left(\eta_{i}\right)\right| \cdot \sum_{j}\left\|\mathfrak{B}_{j-n}^{\vec{\eta}, \vec{s}}\right\|_{1} d \eta d s \\
\lesssim & \sum_{n \geq 100}\left(2^{s_{1} n}+2^{s_{2} n}\right)\|f\|_{1},
\end{aligned}
$$

where

$$
s_{1}=-\varepsilon_{0}+\gamma+2\left(\left[\frac{d}{2}\right]+1\right) \gamma+\delta^{-1} \tau, \quad s_{2}=-\kappa+\varepsilon_{0} .
$$

Now we choose $\tau>\frac{1}{n}$ and $0<\delta^{-1} \tau \ll \gamma \ll \varepsilon_{0} \ll \kappa \ll 1$ such that

$$
\max \left\{s_{1}, s_{2}\right\}<0 \text {. }
$$

Therefore the above sum is convergent and we finish the proof of Lemma 2.5.

\section{Acknowledgements}

The work is supported by NSFC (Nos. 11871096, 11801118 and No. 11571160), China Postdoctoral Science Foundation (No. 2017M621253 and No. 2018T110279) and the Fundamental Research Funds for the Central Universities.

\section{References}

[1] J. Bergh and J. Löfström, Interpolation Spaces, Springer-Verlag, Berlin, 1976.

[2] A. Bressan, A lemma and a conjecture on the cost of rearrangements, Rend. Semin. Mat. Univ. Padova, 110 (2003), 97-102.

[3] A. P. Calderón, Commutators of singular integral operators, Proc. Nat. Acd. Sci. USA, 53 (1965), 1092-1099.

[4] A. P. Calderón, Commutators, singular integrals on Lipschitz curves and application, Proc. Inter. Con. Math. Helsinki, 1978, 85-96, Acad. Sci. Fennica, Helsinki, 1980.

[5] M. Christ and J. Journé, Polynomial growth estimates for multilinear singular integral operators, Acta Math., 159 (1987), 51-80.

[6] Y. Ding and X. Lai, Weighted bound for commutators, J. Geom. Anal., 25 (2015), 1915-1938. 
[7] Y. Ding and X. Lai, On a singular integral of Christ-Journé type with homogeneous kernel, Canad. Math. Bull., 61 (2018), 97-113.

[8] Y. Ding and X. Lai, Weighted weak type $(1,1)$ estimate for the Christ-Journé type commutator, Science China Math., 61 (2018), 517-534.

[9] Y. Ding and X. Lai, Weak type $(1,1)$ bound criterion for singular integral with rough kernel and its applications, Trans. Amer. Math. Soc., 371(3) (2019), 1649-1675..

[10] E. Fabes, M. Jodeit and N. Riviére, Potential techniques for boundary value problems on $C^{1}$-domains, Acta Math., 141 (1978), 165-186.

[11] L. Grafakos, Classic Fourier Analysis, Graduate Texts in Math., 249 (Third edition), Springer, New York, 2014.

[12] L. Grafakos and P. Honzík, A weak-type estimate for commutators, Inter. Math. Res. Not. 20 (2012), 4785-4796.

[13] M. Hadžić, A. Seeger, C. Smart and B. Street, Singular integrals and a problem on mixing flows, 2017. Annales de l'Institut Henri Poincare/ Analyse non lineaire, 35(4) (2018), 921943.

[14] S. Hofmann, Boundedness criteria for rough singular integrals, Proc. London. Math. Soc., 3 (1995), 386-410.

[15] C. Kenig, Harmonic Analysis Techniques for Second Order Elliptic Boundary Value Problems, CBMS Regional Conference Series in Mathematics, 83, Published for the Conference Board of the Mathematical Sciences, Washington, DC, 1994.

[16] X. Lai, Multilinear estimates for Calderón commutators, (2019), Doi: 10.1093/imrn/rny197.

[17] A. Seeger, Singular integral operators with rough convolution kernels, J. Amer. Math. Soc., 9 (1996), 95-105.

[18] A. Seeger, A weak type bound for a singular integral, Rev. Mat. Iberoam., 30 (2014), 961-978.

[19] A. Seeger, C. Smart and B. Street, Multilinear singular integral forms of Christ-Journé type, Memoirs Amer. Math. Soc., 257(1231) (2019). 